\title{
Clinical and morphological manifestation of spontaneous Coxiella burnetii infection in sheep
}

\author{
Rashid Sitdikov, Orazali Mullakaev, and Irina Tyaglova* \\ Kazan State Academy of Veterinary Medicine, Kazan 420029, Russia
}

\begin{abstract}
The study that simultaneously employed negative staining and ultra-thin sectioning to investigate the causative agent of $\mathrm{Q}$ fever in phase I showed that the epizootic strain exhibited morphological characters typical of C. burnetii. Investigation of the ultra-thin structure of sections and whole racketetia cells of the epizootic strain revealed clearly expressed structural basis of the elements of their arrangement and partial similarity of the strain to the reference strains. In animals infected with spontaneous $\mathrm{Q}$ fever, the clinical course of the disease was characterized as severe with signs of intermittent fever up to $42{ }^{\circ} \mathrm{C}$, symptoms of fatigue, decreased food excitability, inactivity, catarrhal rhinitis and conjunctivitis, and cyanosis of mucous membranes. Specific, nonspecific and immunopathological processes developed in organs and tissues: specific changes were characterized by formation of epithelioid cell granulomas with Pirogov-Langhans giant cells in the lymph nodes; nonspecific changes manifested through hyperplastic, macrophage and plasma-cell reactions in lymphoid organs; immunopathological changes were characterized by indurated and fibrotized reticular stroma of lymphoid organs, plasmorrhagia, erythrodiapedesis, mucoid and fibrinoid swelling of the blood vessel walls of the microvasculature, formation of lymphoid and histiocytic infiltrates in the lungs, myocard, liver and kidneys, and dystrophic and necrotic changes in the parenchymal elements of these organs, which cumulatively indicated a morphological manifestation of an allergic reaction of DSR type. The study aimed to investigate some issues of the pathogenesis and pathomorphology of spontaneous $\mathrm{Q}$ fever infection in sheep.
\end{abstract}

\section{Introduction}

It has been 85 years since the outbreak of fever of unknown nature among workers in the massacre in Brisbane (Australia). E.Kh. Derrick's report was a classic study that showed the origin of the name of $\mathrm{Q}$ infection, where $\mathrm{Q}$ means question. Most of its clinical symptoms were described in his report [1-3].

Some symptoms of $\mathrm{Q}$ fever are similar to those of typhoid and typhus fever. Its course can be similar to that of nervous malignances, brucellosis, influenza, and other diseases. Scientists Derrick and Burnet described this pathogen as Ricketsia burnetii, but unlike other rickettsia, it differed in intracellular reproduction, size, polymorphism and sensitivity to antibiotics [9].

The causative agent of $\mathrm{Q}$ fever belongs to the family Rickettsiaceae from the genus Coxiella Philip and the type-species Coxiella Burnetii [12].

$\mathrm{Q}$ fever (coxiellosis) as an anthropozoonotic disease is a serious national economic problem. Its significance becomes obvious due to numerous natural foci of infection, formation of secondary anthropurgic foci, resistance of the pathogen to environmental factors, various transmission routes, and high sensitivity of nonimmune humans and animals to coxiella.
Over the recent years, scientists from Kazan State Academy of Veterinary Medicine and VNIVI focused on the study of the epiotology, clinic, pathogenesis and diagnosis of $\mathrm{Q}$ fever. However, this infection remains unknown to the public and even to the scientific community [3-8].

Immunobiological tests showed 'phase variability' of C. Burnetii. These phases differ in serological patterns. Infection of guinea pigs with C. Burnetii in phases I and II caused production of antibodies to both phases; antibodies to the phase II-antigen appeared first, then antibodies to the phase I-antigen were formed. In nature, C. Burnetii exists in phase I; re-passage of microbes in phase I through chicken embryos cause transition to phase II. Injection of C. Burnetii in phase II to guinea pigs, mice and other animals causes reversal to phase I.

We conducted the electron microscopic study of C. Burnetii of the reference Grita strain and epizootic Barabinsky strain in phase I, and showed the similarity of the ultrastructure of the cell walls and the coxiella cytoplasm regardless of the strain.

However, clinical and morphological studies in sheep with epizootic diseases were not studied. The aim of our study was to investigate the clinical and morphological picture of spontaneous C. Burnetii infection in sheep [9$11]$.

Corresponding author: i.tiaglowa@yandex.ru 


\section{Material and Methods}

We studied sheep killed in a failed farm due to incidence of $\mathrm{Q}$ fever. Animals were sacrificed for diagnostic purposes. Intravital serological studies revealed specific complement binding antibodies to the agent of $\mathrm{Q}$ fever in a titer of 1:40 in the blood serum of these animals. The presence of specific antibodies to the agent of $\mathrm{Q}$ fever in the blood serum of the infected animals was determined by the prolonged complement fixation test using the method by R.G. Gosmanova and R.Kh. Yusupova (1984). Rickettsial antigen in lymphoid organs and tissues was detected by direct and indirect immunofluorescence methods by Coons. Pieces of lymph nodes, spleen, kidneys, heart, lungs and brain were taken for pathological, histological and immunofluorescence studies. Tissue sections for histological examination were fixed in alcohol-formalin 9:1 and then processed by generally accepted methods. Sections of the spleen and lymph nodes were used for electron microscopic studies. Tissue was treated by generally accepted electron microscopy techniques:

- negative staining in phosphoric tungsten acid (pH 7.0) at concentrations of $0.15,1,2$ and $5 \%$ exposed for 1 minute, 45 seconds, 30 seconds, 15 and 5 seconds and saturation with solutions of uranyl acetate a) aqueous, exposed for 3 minutes, 1 minute and 30 seconds; b) $70 \%$ ethanol, exposed for 3 minutes, 1 minute and 30 seconds;

- ultra-thin sectioning. In this case, the test material was pelleted until a solid 'cake', the dry material was poured into agar to cut pieces for further processing. The material was fixed in $3 \%$ glutaraldehyde solution for 2 hours at $+4{ }^{\circ} \mathrm{C}$, and then it was fixed with osmium tetroxide, dehydrated and poured into a mixture of epoxy resins.

Sections were made using an LKB-3 ultratome, examined using a JEM-100B electron microscope at $\mathrm{x}$ 15,000-50000 instrumental magnification. Photographic plates designed for nuclear research were used to take pictures of the material. The plates were marked and electron diffraction patterns were printed.

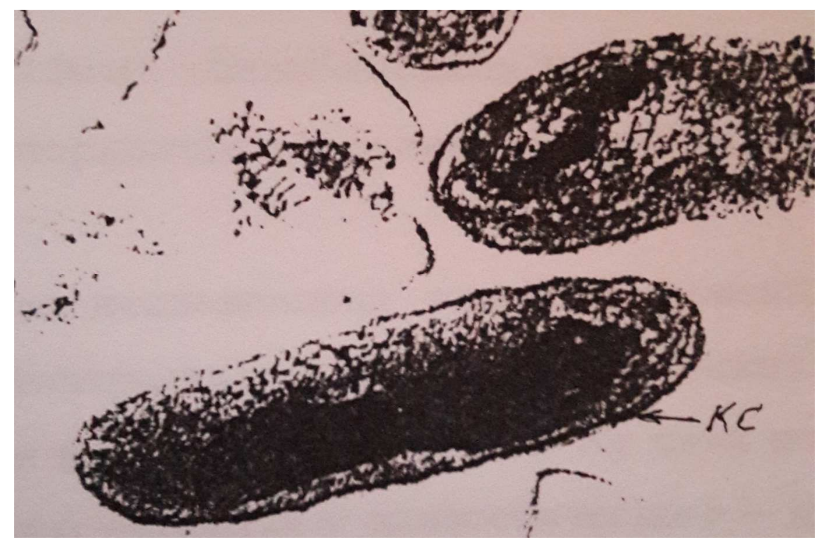

Fig. 1. C. Burnetii in phase I. Ultra-thin section, $\times 50,000$

Simultaneous use of negative staining and ultra-thin sectioning to study the submicroscopic arrangement of C. Burnetii provided data on the shape, characteristic structural features, surface and internal structures of the cells of the studied strain. The studies showed that the strain exhibited morphological characters of phase I typical of Coxiella burnetii (Fig. 1).

Bacteriological studies were carried out in accordance with the current Rules for the veterinary inspection of slaughter animals and veterinary and sanitary examination of meat and meat products. GOST 218375-75. Meat. Methods of bacteriological analysis.

\section{Results}

Simultaneous use of negative staining and ultra-fine sectioning to study the submicroscopic arrangement of C. Burnetii isolated from infected animals provided data on the shape, characteristic structural features, surface and internal structures of the cells of the studied strains [5].

The studies showed that the strains had morphological characters of phase I typical of $\mathrm{C}$. Burnetii. They were $800-1000 \mathrm{~nm}$ in size. The bodies are not uniform in shape, rod-shaped and spindle-shaped, club-shaped bodies and sometimes bodies with bizarre contours were found in the strains. Negative staining revealed a strongly folded surface of C. Burnetii. Ultrafine sections displayed C. Burnetii of an oval shape and smaller sizes: 780-900 $\mathrm{nm}$.

The cell wall consisted of three layers: two osmiophilic layers separated by the osmiophobic layer. The total cell wall thickness was 8.5-9 nm. It could be smooth with no deep folds. A three-layer plasmolemma 7-7.5 nm thick was adjacent to the cell wall from the inside. The electron density and granularity of the rickettsioplasma was moderate. A spindle-shaped electron-dense nucleoid formed of spirally coiled chromatin strands is found in the electrontransparent vacuole along the longitudinal axis, sometimes in an eccentric position. The shape of the nucleoid is not always strictly spindle-shaped, sometimes club-shaped thickenings could be observed at one of its ends. In addition, randomly arranged electrondense inclusions could be observed throughout the rickettsiaplasma in some coxiella.

In animals infected with spontaneous $\mathrm{Q}$ fever, body temperature increased to $40.8-42{ }^{\circ} \mathrm{C}$. Repeated relapses were also observed. During the rise in body temperature, the animals exhibited complete refusal of food, depressed state, inactivity, sometimes diarrhea, catarrhal rhinitis, conjunctivitis, cyanosis of the mucous membranes of the nasal and oral cavities.

An autopsy study of carcasses of animals showed a 1.5-2-fold increase in lymph nodes, the sections of individual lymph nodes were reddish in color. The spleen was enlarged 1.5 times, the section had a welldefined follicular pattern, the liver was slightly increased in size, tan, the gall bladder was filled with bile. Histological examination showed that lymphoidproliferative and magrophagic reactions resulted in depletion of cellular elements of the cortical substance of the lymph nodes, follicles, and periarterial spleen sleeves in lymphoid organs [4]. 
Germinal centers with sparse arrangement of cellular elements could be clearly observed in the lymph nodes. Blast cells and lymphocytes were mostly found in the centers. Lymphoid tissue was moderately hyperplastic, and the reticular fibers were slightly roughened. The lymph sinuses were enlarged and contained a significant number of lympho- and plasmoblasts, and macrophages with fine-grained granules of reddish-brown pigment.

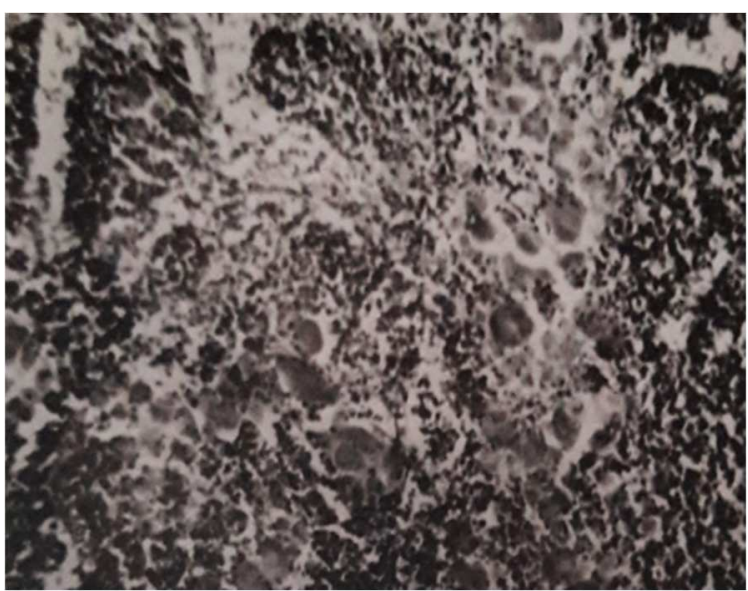

Fig. 2. Epithelioid cell granuloma with giant cells in the lymph node, stained with hematoxylin and eosin, $\times 100$

Small epithelioid cell granulomas were found in the cortical layer of the node. The granulomas were lighter in shade as compared with the surrounding tissues (Fig. 2).

Numerous epithelioid cells were scattered throughout the node, which displayed diffuse emerald fluorescence of four crosses during processing of the sections with a specific luminescent serum. Sinus macrophages, many of which were increased in volume, also desplayed positive immunofluorescence (Fig. 3).

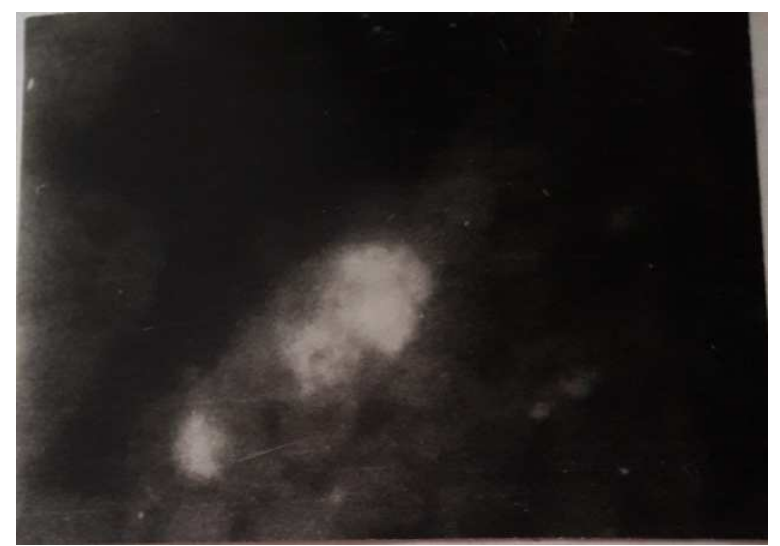

Fig. 3. Specific luminescence of coxiella antigen in the macrophage of a sheep's lymph node. Coons' direct immunofluorescence method, $\times 900$

The trabecular base of the organs was edematous with some areas of connective tissue base dissociation and lymphoid-histiocytic cell infiltration. When stained according to the technique by Romanovsky-Giemsa, infiltration of the connective tissue base of lymph nodes by mast cells and their degranulation were revealed. The vessel walls of the organ exhibited signs of mucoid swelling, the endothelium was swollen, vacuolated, desquamated.

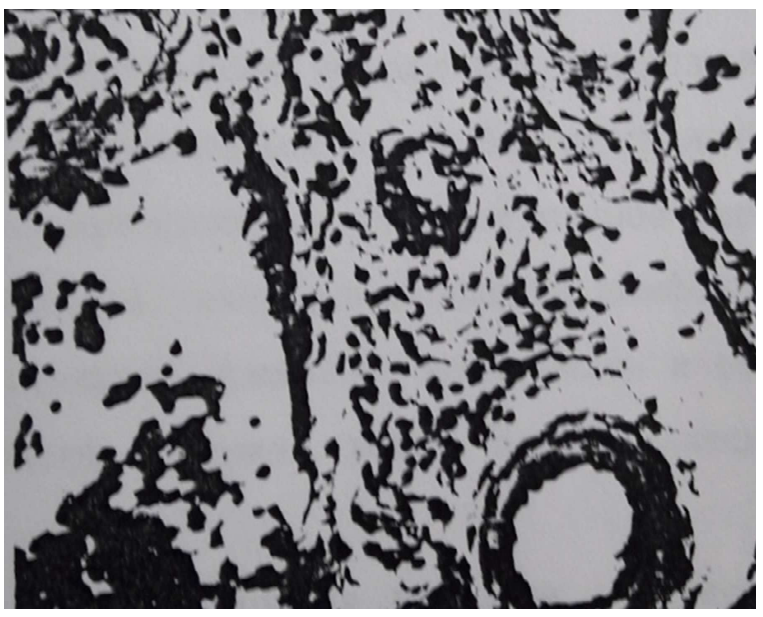

Fig. 4. Edema, dissociation and cell infiltration of the connective tissue base of trabeculae in the deep inguinal lymph node, stained by hematoxylin and eosin, $\times 400$

In the lungs of sheep infected with spontaneous $Q$ fever, specific luminescence was noted for alveolar macrophage [6], which were swollen and displayed four cross fluorescence (Fig. 5).

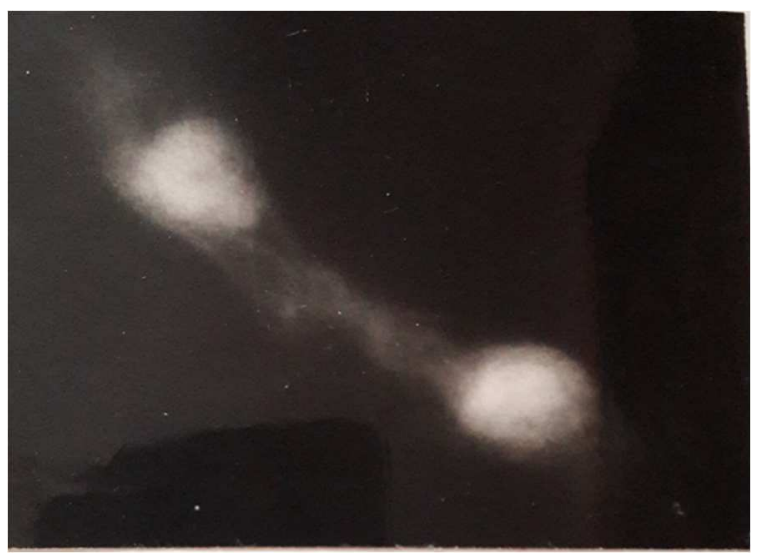

Fig. 5. Luminescence of alveolar macrophages of the infected sheep. Coons' indirect immunofluorescence method, $\times 900$

Histological examination showed focal thickenings of the interalveolar septa due to blood filling of the capillaries and proliferation of lymphoid-histiocytic cells. Many alveoli contained serous exudate with alveolar macrophages, histiocytes, and single neutrophils. A small amount of red blood cells was observed in the lumen of some alveoli. The connective tissue base of blood vessels and bronchioles was infiltrated by lymphoid-histiocytic cells with subsequent formation of cell couplings [8].

In kidneys, the pattern of the structure is preserved. Some renal corpuscles with multiplied capillary network exhibited proliferative membranous glomerulonephritis. The capsule lumen was enlarged in many renal corpuscles; accumulations of a small amount of protein fluid, occasionally mixed with cellular elements, were found in corpuscles. 
The convoluted tubule epithelium contained a significant amount of protein mass. In the interstitium of the organ and along the vessels, small-sized round-cell infiltrates were observed, which sometimes formed couplings around the vessels. The wall of many vessels showed mucoid and fibrinoid swelling. The vessels of the pyramids are moderately full-blooded. The immunofluorescence study of the kidney tissue revealed positive luminescence of the $\mathrm{Q}$ fever antigen in vascular glomeruli in the form of a diffuse two cross fluorescence.

In the liver, plethora of the central veins and sinusoidal capillaries could be observed. Hepatocytes exhibited signs of granular, sometimes fatty degeneration. Unequal plethora of sinusoids and parenchymal dystrophy caused discompletion of the beam-like structure of the organ. Stellate reticuloendotheliocytes were proliferated and swollen, many of which had a foamy cytoplasm and were often found in the lumen of sinusoids. The study of the organ tissue by the method of fluorescent antibodies showed a bright diffuse four cross fluorescence of these cells. Focal accumulations of lymphoid-histiocytic cells were found perivascularly.

Cardiomyocytes exhibited granular dystrophy. Interstitial tissue was swollen and fibrous. Hyperemia of the capillary network was noted. The wall of the network was mucoid swollen. The fibers of the conductive system were swollen and loose.

In the brain of the infected animals, vascular congestion, edema of the brain substance, perivascular and pericellular edema (Fig. 6) and non-purulent lymphocytic encephalitis could be clearly observed. In addition, perivascular infiltrates from lymphoid cells, which generally formed pronounced perivascular couplings, were noted (Fig. 7).

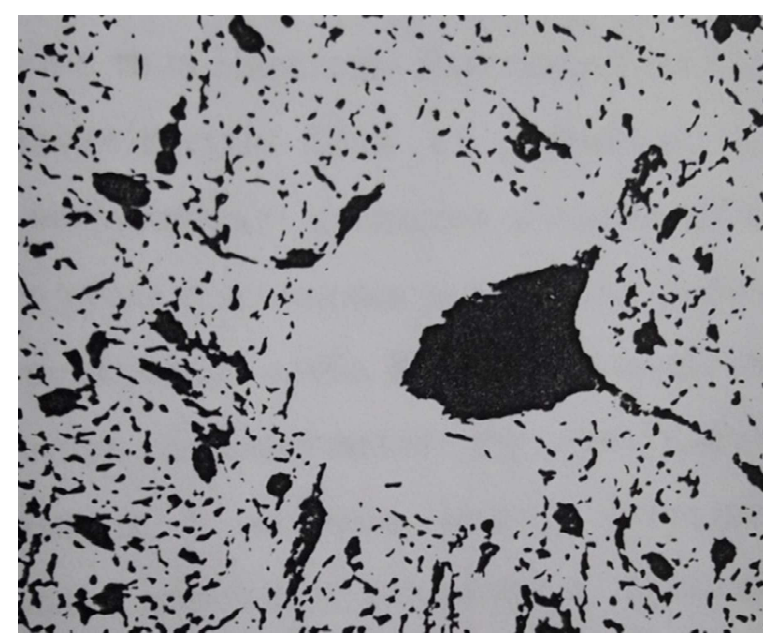

Fig. 6. Pericellular edema of brain cells stained with hematoxylin and eosin, $\times 400$

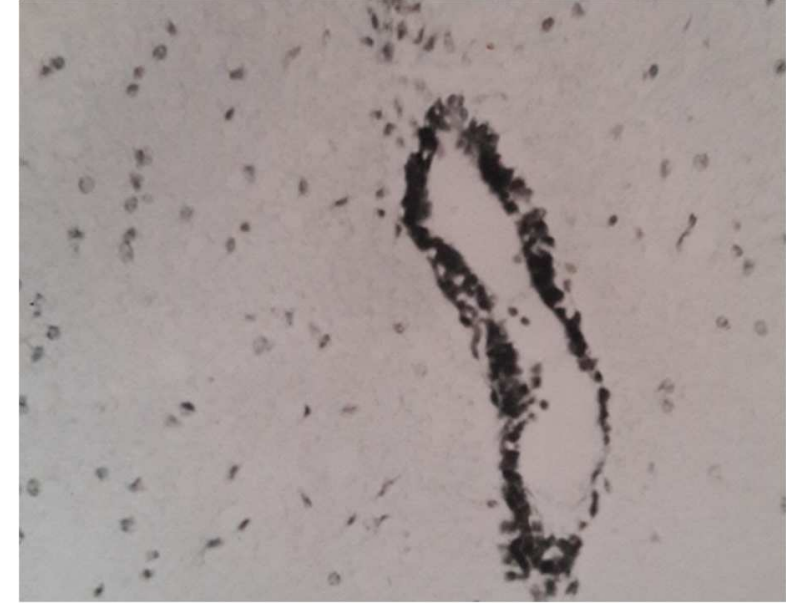

Fig. 7. Perivascular lymphoid coupling in the sheep brain stained with hematoxylin and eosin, $\times 200$

\section{Conclusion}

Thus, electron microscopic methods used in the study showed C. Burnetii (Q-Fever) in phase 1 in the animals infected with spontaneous Q-fever isolated from sheep living in the sheep farm with incidence of O-fever. The infected animals exhibited a severe clinic of the disease, which was characterized by intermittent fever up to $42{ }^{\circ} \mathrm{C}$, symptoms of fatigue, decreased food excitability, inactivity, catarrhal rhenitis and conjunctivitis, and cyanosis of mucous membranes.

Specific, nonspecific and immunopathological processes developed in organs and tissues of infected animals: specific processes implied the formation of epithelioid cell granulomas with Pirogov-Langhans cells giant in the lymph nodes; nonspecific changes manifested hyperplastic, macrophage, plasma cell reactions in lymphoid organs; immunological processes were characterized by indurated and fibrotized reticular stroma of lymphoid organs, plasmorrhagia, erythrodiapethesis, mucoid and fibrinoid swelling of the blood vessel walls of the microvasculature, by the formation of lymphoid histiocytic infiltrates in the lungs, myocard, kidney and liver, and by necrotic changes in the parenchymal elements of these organs, which indicated a morphological manifestation of an allergic reaction such as delayed skin reaction.

\section{References}

1. K.M. Loban, Q fever (Moscow, 1987)

2. K.M. Loban, Yu.V. Lobzin, E.P. Lukin, Human Rickettsioses: A Guide for Physicians (Moscow; St. Petersburg, 2002) pp. 393-474

3. R.I. Sitdikov, N.Z. Shigapov, Pathomorphology and some issues of the pathogenesis of $Q$ fever experimentally induced in sheep. Issues of rickettsiology, in: Proc. of the IV All-Union Conf. Actual issues of epidemiology, diagnosis and treatment, prevention of rickettsiosis (1989) pp. 2931 
4. R.I. Sitdikov, Pathomorphology and pathogenesis of $Q$ fever in animals upon infection with different strains of Burnet's coxiella and in a joint course with radiation sickness, Dissertation (Kazan, 2000) $35 \mathrm{p}$.

5. V.A. Shoshokin, R.Kh. Yusupov, R.G. Gosmanov, Electron microscopic examination of the state of the causative agent of fever $Q$ in the organs of infected animals, in: Problems of infectious and noncontagious pathology of agricultural animals in the Proc. of the All-Russ. Sci. and Methodol. Conf. on Patholog. Anatomy of Agricult. Animals (1998) pp. 118-122

6. N.Z. Shigapov, Histological and immunofluorescence studies of organs of sheep with spontaneous $Q$ fever, in: To livestock - a comprehensive development program: Abstracts of the Republ. Sci.-Indust. Conf. (Kazan, 1990) p. 52

7. N.F. Fetisova, M.T. Gafarova, Ecological and epidemiological aspects of coxiellosis, RAMS Bull., 7, 15-18 (2008)
8. R.Kh. Yusupov, R.G. Gosmanov, V.A. Shoshokin, Immunomorphological changes in organs and tissues of animals with experimentally induced $Q$ fever, in: Proc. of the Republ. Sci.-Indust. Conf. on Actual Probl. of Veter. Med. and Zootech. (1997) p. 42

9. O.G. Baca, D. Paretsky, $Q$ fever and Coxiella burnetii: A model for Host-Parasite Interactions, Microbiol. Rev. (1983)

10. I. Galperin, J.M. van Dljk, Chronic $Q$ fever hepatitis, Isr. Med. Assoc. J., 7(8), 529-30 (August 2005)

11. A. Medic, B. Dzelalija, P.V. Punda, M.I. Gjenero, V. Gilic, $Q$ fever epidemic among employees in a factory in the suburb of Zadar, Croatia, Croat. Med. J., 46(2), 315-319 (April 2005)

12. C.B. Philip, Nomenclature of the pathogenic rickettsiae, Am. J. Hyg., 37, 301-309 (1943). 